\title{
An Optimized Real Time Image Codec for Image Data Transmission and Storage
}

\author{
Nirmala Salam \\ CDAC-Mumbai \\ Gulmohar Cross Road No.9 \\ Juhu, Mumbai -49, India
}

\author{
Rekha Nair \\ CDAC-Mumbai \\ Gulmohar Cross Road No.9 \\ Juhu, Mumbai -49, India
}

\begin{abstract}
The need for an efficient technique for compression of Images is ever increasing because the raw images require large amount of disk space and large amount of time for images to be sent over the internet or downloaded from the web pages which seems to be very big disadvantage during transmission $\&$ storage. In this paper, we propose a simple image compression scheme to obtain better reconstructed image on decompression. The scheme is mainly based on DCT (Discrete Cosine Transform), which is one of the well known lossy image compression techniques. Our approach does not involve any encoding or decoding method like other conventional compression methods thereby decreasing the time complexity of overall process.. The proposed scheme is simple, efficient and has low computational cost and high compression ratio and thus satisfying the current prime requirement of image data transmission and storage.
\end{abstract}

\section{General Terms}

Digital Image processing, Image Codec

\section{Keywords}

Color Image compression; grayscale image compression, Decompression of image, DCT, LBG, Vector Quantization.

\section{INTRODUCTION}

Due to the increasing demand on data transfer and storage image compression has been an important research area for many years. With the advent of digital cameras, one of the most common uses has been the storage, manipulation, and transfer of digital images. The files that comprise these images, however, can be quite large and can quickly take up precious memory space on the computer's hard drive. A gray scale image that is $256 \times 256$ pixels have 65,536 elements to store and a typical $640 \times 480$ color image have nearly a million! The size of these files can also make downloading from the internet a lengthy process. So, here comes the need of image compression which reduces the amount of data required for representing sampled digital images and therefore reducing the size and the cost for storage and transmission [1].

Image compression may be lossy or lossless. Lossless compression is preferred for archival purposes and often for medical imaging, technical drawings, clip art, or comics. This is because lossy compression methods, especially when used at low bit rates, introduce compression artifacts. Lossy methods are especially suitable for natural images such as photographs in applications where minor (sometimes imperceptible) loss of fidelity is acceptable to achieve a substantial reduction in bit rate.
In our proposed algorithm we have used DCT a lossy compression technique for image compression

\section{DCT TECHNIQUE}

The DCT is a technique for converting a signal into elementary frequency components. Like any Fourier-related transform, discrete cosine transforms (DCTs) express a function or a signal in terms of a sum of sinusoids with different frequencies and amplitudes. Like the discrete Fourier transform (DFT), a DCT operates on a function at a finite number of discrete data points. The obvious distinction between a DCT and a DFT is that the former uses only cosine functions, while the latter uses both cosines and sines (in the form of complex exponentials). However, this visible difference is merely a consequence of a deeper distinction: a DCT implies different boundary conditions than the DFT or other related transforms.[3]. DCT is calculated by using (1)

$$
b_{p q}=c_{p} c_{q} \sum_{m=0}^{M-1} \sum_{n=0}^{N-1} a_{m n} \cos \frac{\pi(2 m+1) p}{2 M} \cos \frac{\pi(2 n+1) q}{2 N}
$$

Where $\quad 0 \leq p \leq M-1$ and $0 \leq q \leq N-1$

$$
C_{p}=\left\{\begin{array}{l}
1 / \sqrt{M}, p=0 \\
\sqrt{2 / M}, 1 \leq p \leq M-1
\end{array}\right.
$$

The value bpq are called the DCT coefficients of $\mathrm{M}$ by $\mathrm{N}$

$$
c_{q}=\left\{\begin{array}{l}
1 / \sqrt{N}, q=0 \\
\sqrt{2 / N}, 1 \leq q \leq N-1
\end{array}\right.
$$

matrix A.

\section{IDCT TECHNIQUE}

The DCT is an invertible transform, and its inverse (IDCT) is calculated using (4):

$a_{m n}=\sum_{p=0}^{M-1} \sum_{q=0}^{N-1} c_{p} c_{q} b_{p q} \cos \frac{\pi(2 m+1) p}{2 M} \cos \frac{\pi(2 n+1) q}{2 N}$ 


$$
\begin{gathered}
\text { Where } \begin{array}{l}
0 \leq m \leq M-1 \\
0 \leq n \leq N-1
\end{array} \\
C_{p}=\left\{\begin{array}{l}
1 / \sqrt{M}, p=0 \\
\sqrt{2 / M}, 1 \leq p \leq M-1
\end{array}\right. \\
C_{q}=\left\{\begin{array}{l}
1 / \sqrt{N}, q=0 \\
\sqrt{2 / N}, 1 \leq q \leq N-1
\end{array}\right.
\end{gathered}
$$

The JPEG process is a widely used form of lossy image compression that centers around the DCT. The DCT works by separating images into parts of different frequencies. During a step called quantization, where part of compression actually occurs the less important (higher) frequencies are discarded, hence the use of the term 'lossy'

The conventional compression method involves:

- Applying DCT block wise from left to right and top to bottom.

- Quantize each block by dividing each elements of a matrix by the corresponding elements of a luminance matrix.

- Apply encoding process to the coefficients of quantized matrix converting it into a bit stream

The conventional decompression method involves:

- Apply decoding process to the bit stream and convert it back to the blocks of matrix

- Multiply each elements of a blocks by the luminance matrix you have selected

- Apply IDCT (Inverse Discrete Cosine Transform) to each block

\section{PROPOSED APPROACH}

The proposed approach develops an optimized image codec. On compression side it applies DCT (Discrete Cosine Transform) on image row wise only, using (1). Here we are not applying DCT directly on whole image and neither the block wise application of DCT is done. The IDCT (Inverse DCT) is applied only to the high frequency area of each row using (4). Rest of the samples is padded with zeros.
Normalizing and adjusting the intensity of the resultant matrix compresses the image size to a greater extent. Block diagram of the compression algorithm is shown in Figure 1.

On the decompression side the application of reverse process of above mentioned compression of image on compressed image results into decompressed image which is almost similar to the original image. The Decompression algorithm can used on compressed image obtained in real time or it can be used on the stored compressed image. Both ways it can work as shown in Figure 10.

\section{EXPERIMENTAL RESULTS}

The proposed approach has been tested with the images of varied format and the result was satisfactory. For testing purpose we run compression algorithm on one computer and decompression algorithm on another computer. The quality of the compressed image obtained through our compression algorithm is sufficient to reconstruct the original image using our decompression method with slight loss in quality and size of the compressed image is acceptable to transmit through the low bandwidth network and sufficient for storage. The image size we have used here is of $1440 \times 900$ pixels. Figure 2, 3, 4 and 5 below shows the results of application of above compression and decompression method on the JPEG, BMP, PNG and TIF images. Table 1 below depicts the computational cost of proposed compression and decompression algorithm on JPEG, BMP, PNG and TIF images and Table 2 shows the original and compressed size of the JPEG, BMP, PNG and TIF image on application of our compression algorithm.

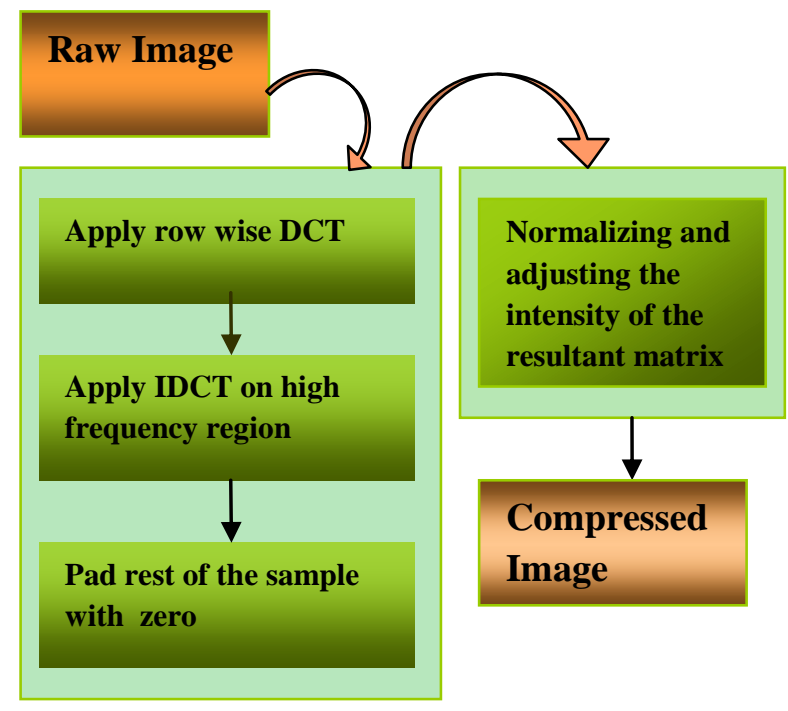

Fig 1: Block Diagram of proposed Approach 


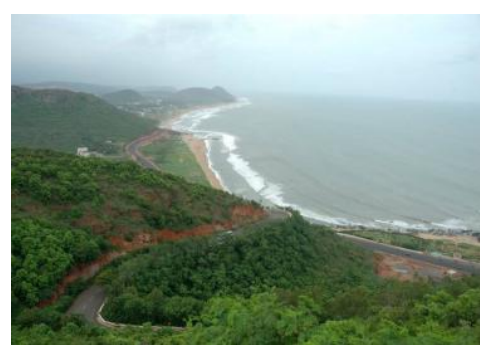

a) Original Image (Scenery)

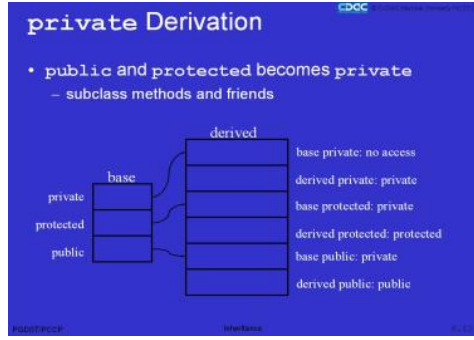

d) Original Image $(\mathrm{C}++$ Slide $)$

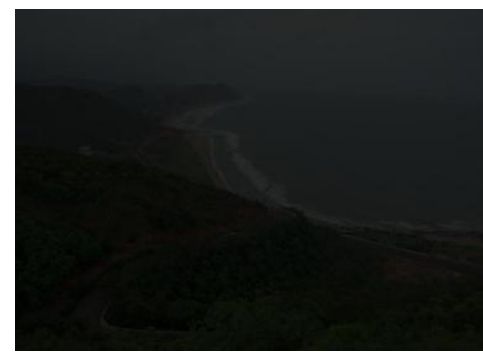

b) Compressed Image

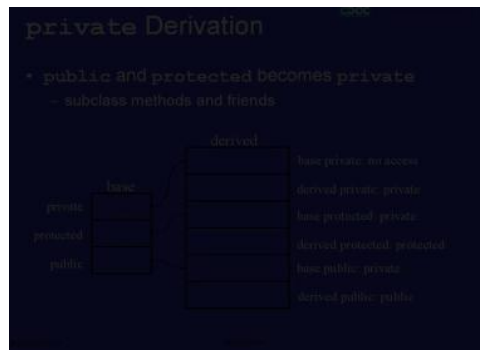

e) Compressed Image

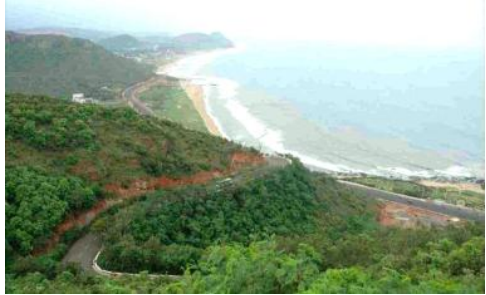

c) Decompressed Image

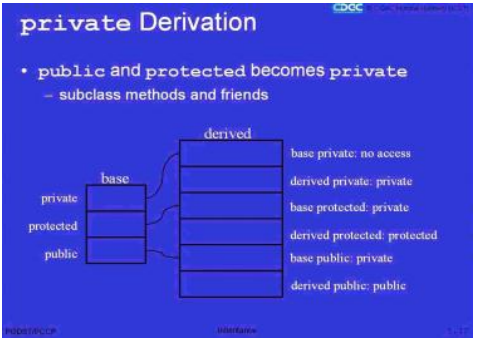

f) Decompressed Image

Fig 2: Compression and Decompression of images using JPEG format 1440x900 Resolution

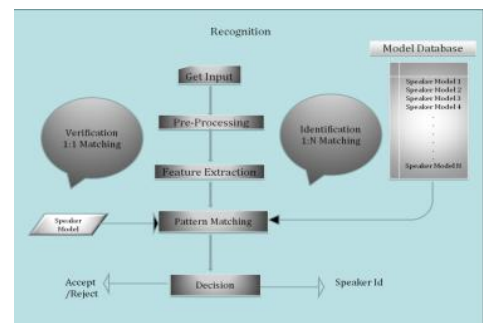

a) Original Image (flowchart)

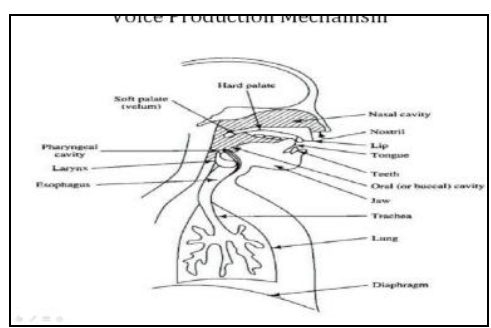

d) Original Image (Voice)

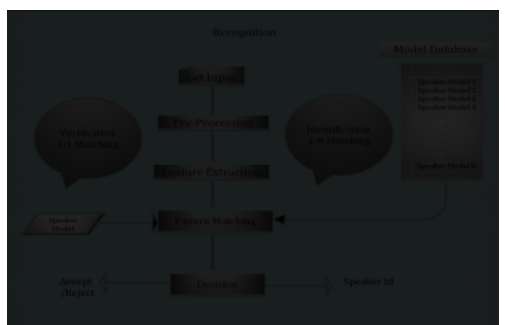

b) Compressed Image

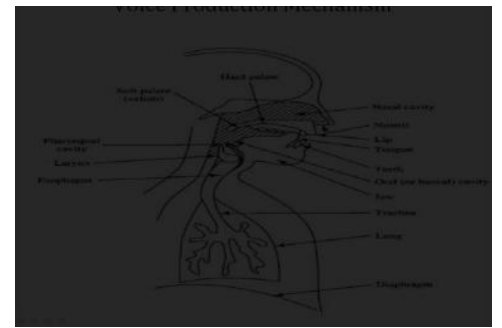

e) Compressed Image

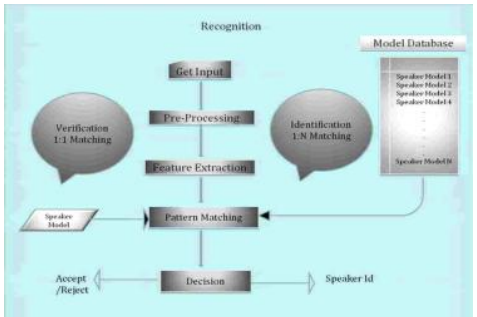

c) Decompressed Image

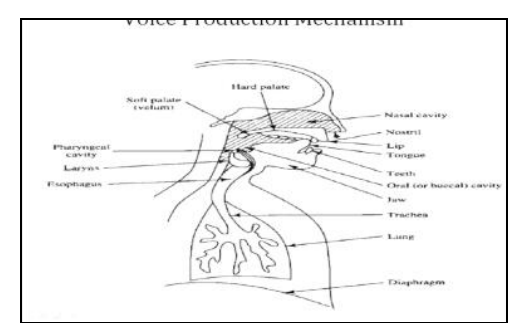

f) Decompressed Image

Fig 3: Compression and Decompression of images using BMP format 1440x900 Resolution

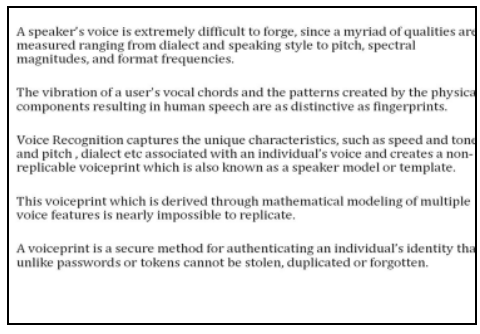

a) Original Image (text slide))

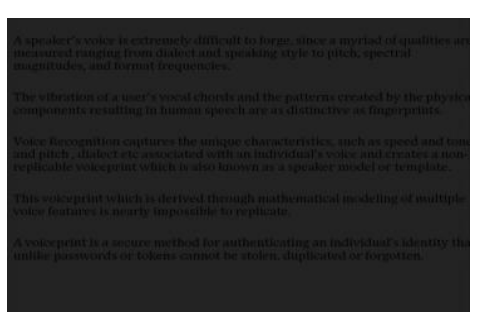

b) Compressed Image

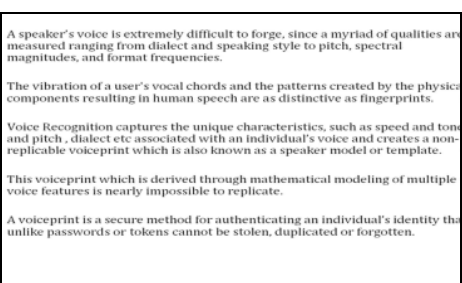

c) Decompressed Image 


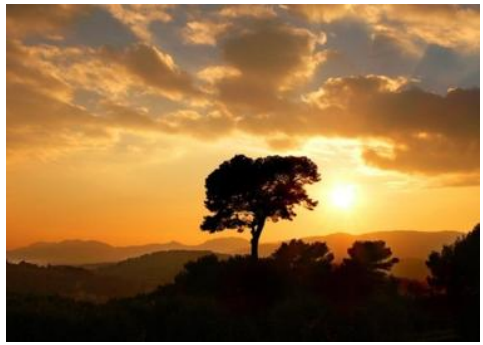

d) Original Image (sunrays)

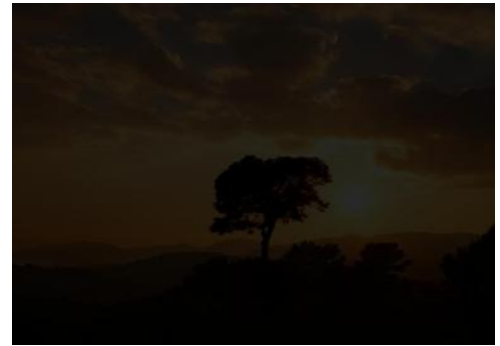

e) Compressed Image

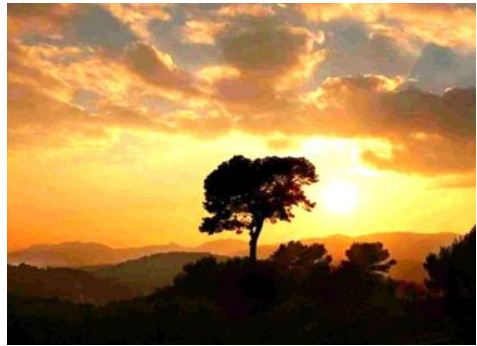

f) Decompressed Image

Fig 4: Compression and Decompression of images using PNG format 1440x900 Resolution

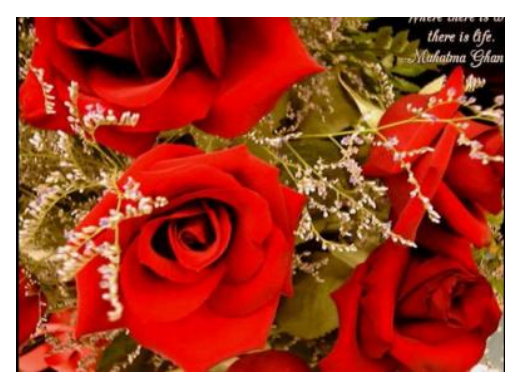

a) Original Image (flower)

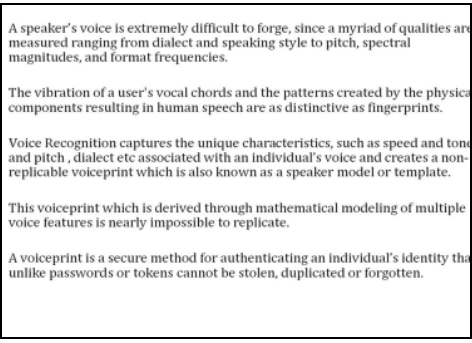

d) Original Image (text slide)

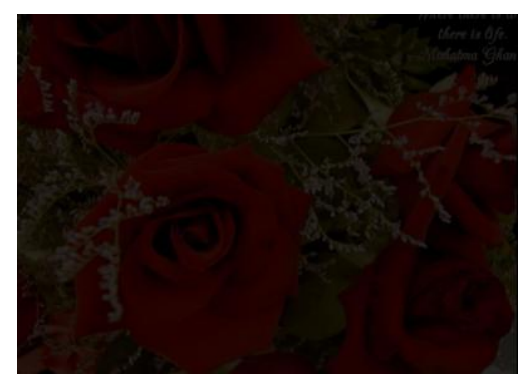

b) Compressed Image

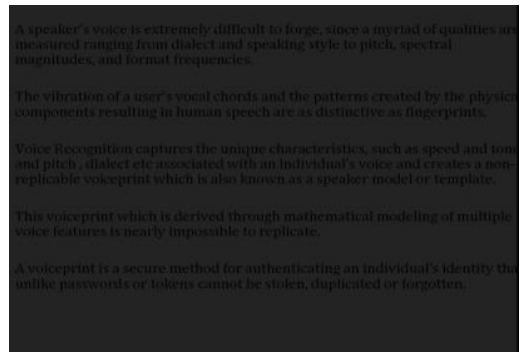

e) Compressed Image

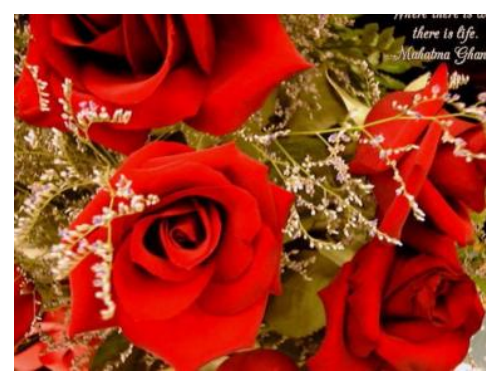

c) Decompressed Image

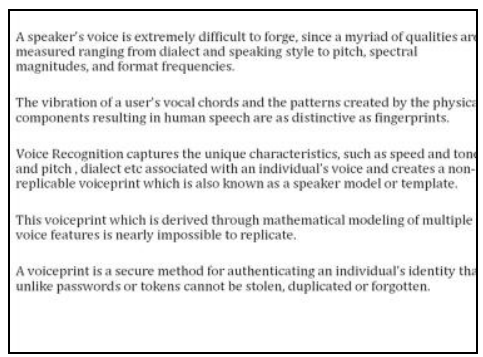

f) Decompressed Image

Fig 5: Compression and Decompression of images using TIF format 1440x900 Resolution 
Table 1. Showing average computational cost of the above images

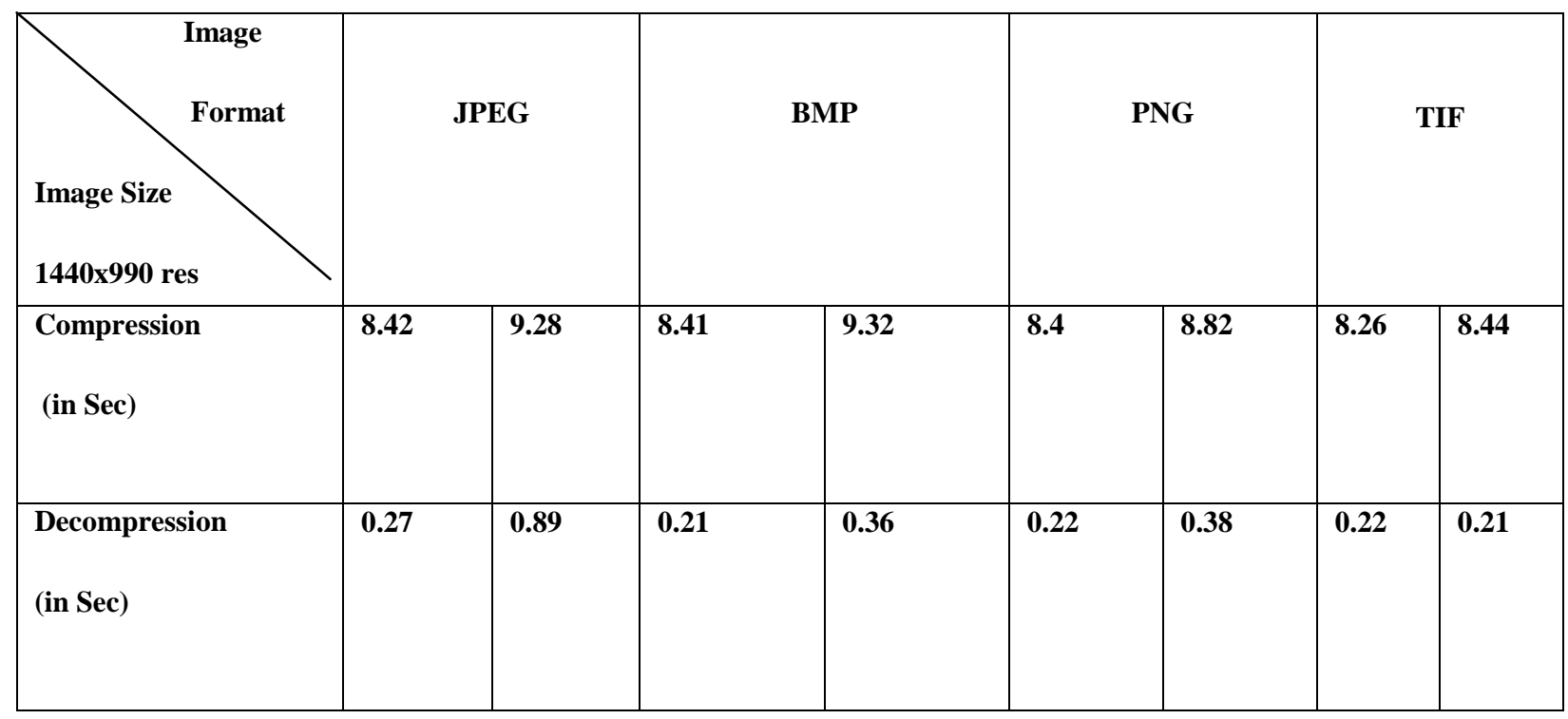

Table 2. Showing original image size and corresponding compressed image size

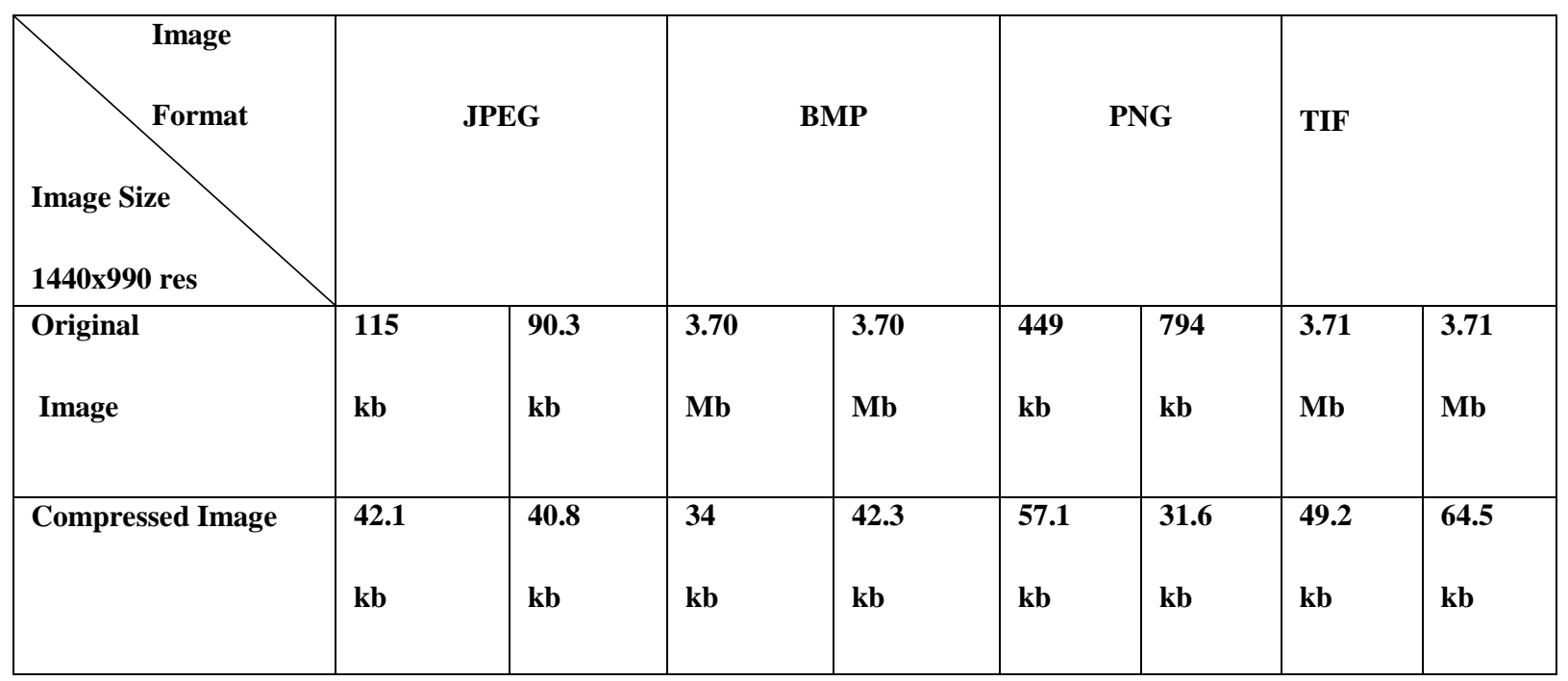




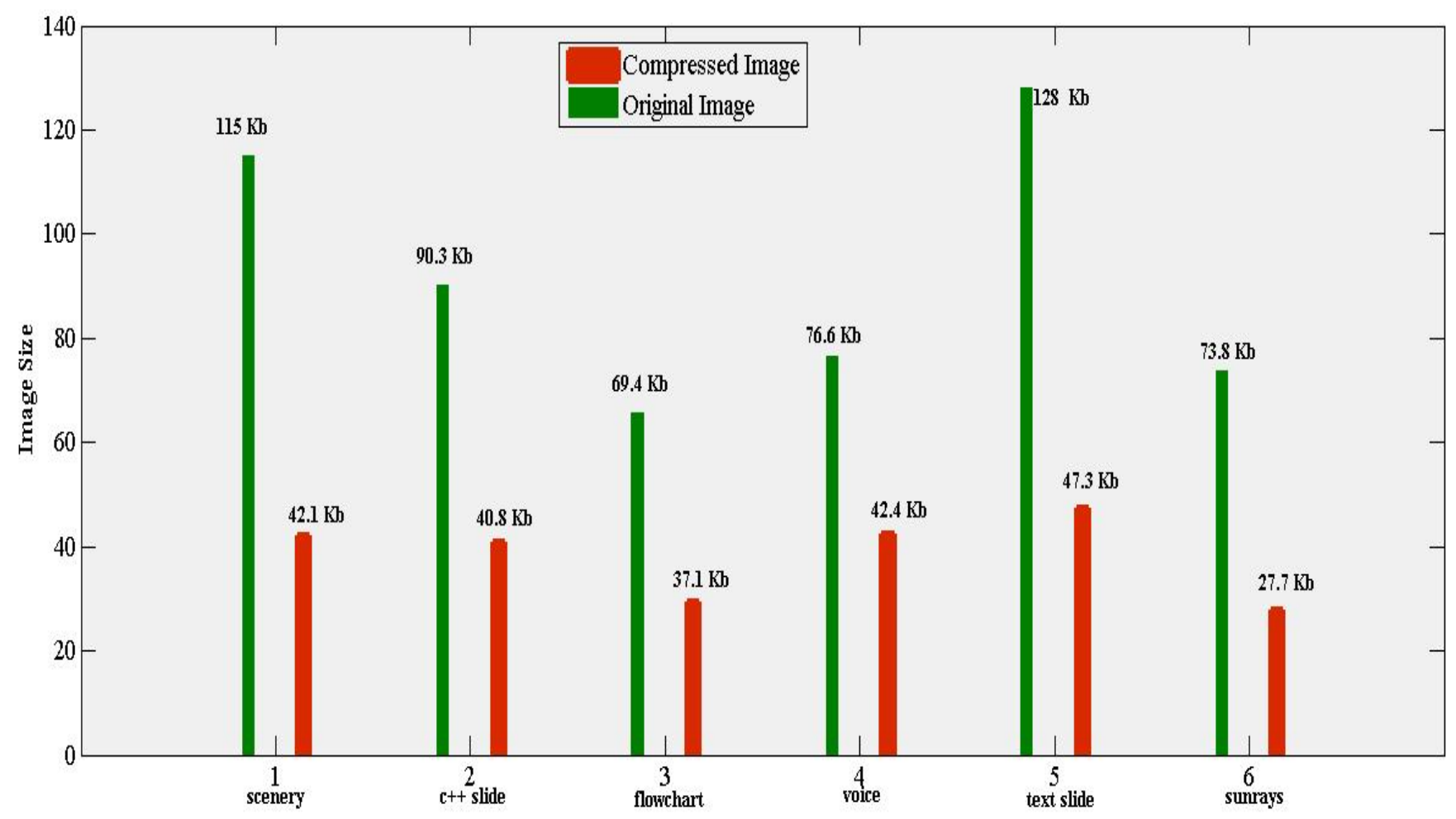

Fig 6: Original Images Vs Compressed Images for JPEG Format

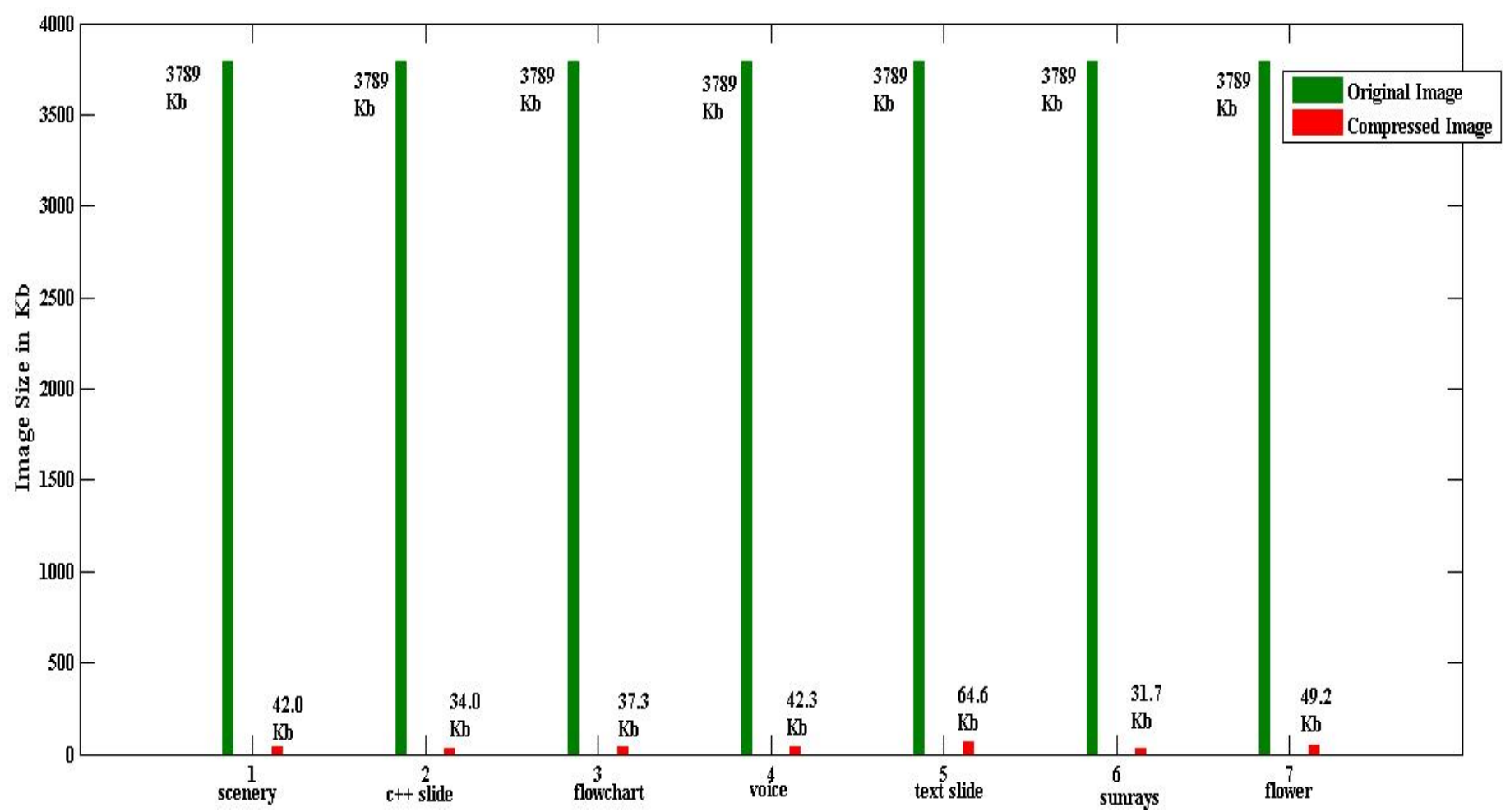

Fig 7: Original Images Vs Compressed Images for BMP Format 


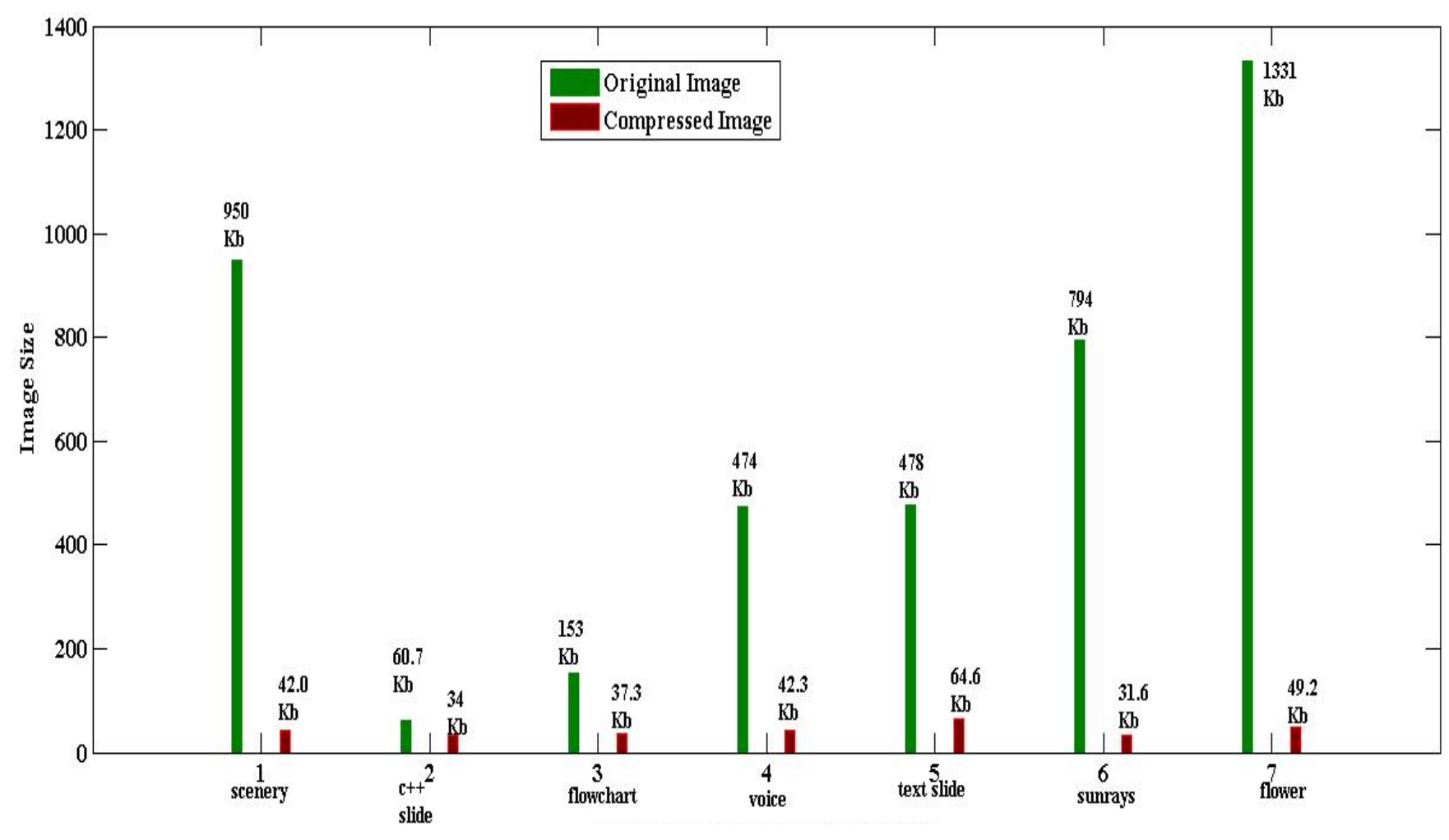

Fig 8: Original Images Vs Compressed Images for PNG Format

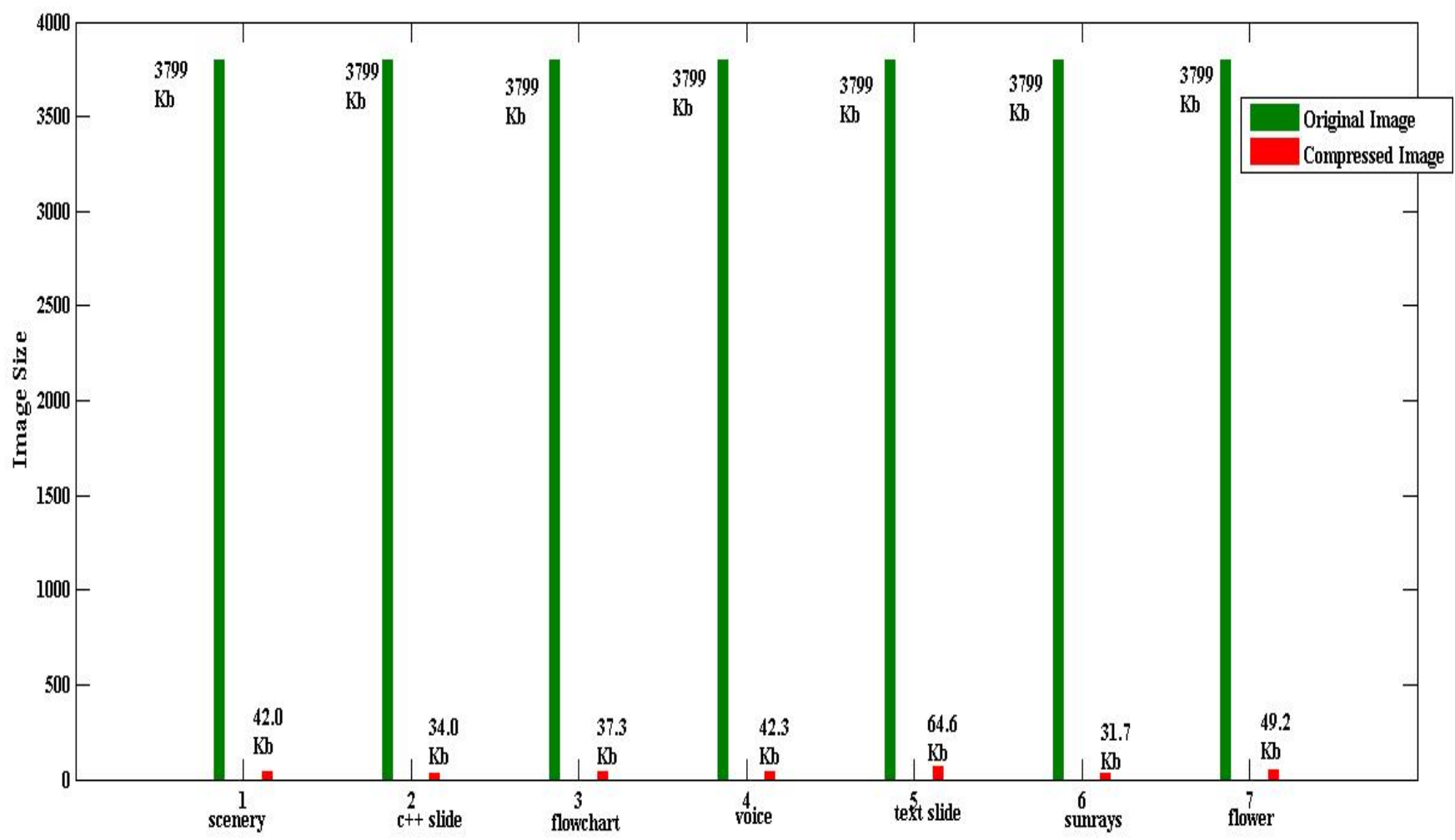

Fig 9: Original Images Vs Compressed Images for TIF Format 


\section{CONCLUSION}

In this paper we have proposed a method for real time compression and decompression of images for transmission through low bandwidth. The PSNR value calculated for the above images is 29.5. The proposed method is able to compress the image data size to a remarkable extent .The reconstructed decompressed image quality is almost similar to the original image quality but is acceptable in terms of storage and transmission. We have tested this algorithm for jpeg, bmp, png and tif format images and result were satisfactory.

\section{ACKNOWLEDGEMENT}

This work is being carried out as a part of Real Time Video Compression and Decompression for eLearning, $R \& D$ project under National eLearning Plan funded by DIT (Department of Information Technology), Government of India. We thank Dr. Padmaja Joshi and Mr. Vinod kumar for their guidance and Mr. Ashwin Nivangune for his support.

\section{REFERENCES}

[1] J.h. Pujar,L.M. Kadlaskar. ,2005-2010. A new lossless method of image compression and decompression using Huffman coding techniques in Journal of Theoretical and Applied Information Technology 2005-2010, Vol 15.No1/3

[2] D.S. Taubman , M.W. Marcellin, JPEG2000 Image Compression, Fundamentals, Standards and Practice. Boston, MA: Kluwer, 2002.

[3] k. Cabeen and P. Gent., Image Compression and Discrete Cosine Transform, Gent, Math 54, College of Redwood

[4] H.S. Malvar, Fast progressive Image Coding Without Wavelets, In Proceedings of Data Compression Conference, Snowbird,UT,PP.243-252, 2000
[5] L. Zhifeng, F. Changhong, F. Xu, Q. Zhicong, W.Shunxiang, 2009, An Easy Image Compression Method and Its Realization Base on Matlab, In Proceedings of ICIECS 2009, Pages 1-4

[6] A.K. Pal, G.P. Biswas and S. Mukhopadhyay, 2010. A Hybrid DCT-VQ Based Approach for Efficient Compression of Color Images. In Proceedings of Int'l Conf. on Computer \& Communication Technology 2010, Pages 177-181

[7] H.S.Malvar, Fast Encoder for bi-level Images, In proceedings of Data Compression Conference, Snowbird, UT,PP, 253-262, 2001

[8] T.J.Chen, K.S. Chuang, 2010. Pseudo Lossless Image Compression Method, In Proceedings of 3rd International Congress on Image and Signal Processing (CISP2010)

[9] C. Martinez, 2006. An ACO algorithm for image compression, In clei electronic journal, volume 9, number 2, paper 1, december 2006

[10] L.T.W. Alexander, P. Morgan, R.A. Young, A Gaussian Derivative Based Version of JPEG for Image Compression and Decompression, In IEEE Transaction on Image Processing, vol 7, no. 9, pp. 1311-1320, September, 1998

[11] I.Vilovic, An Experience in Image Compression using Neural Networks, In $48^{\text {th }}$ International Symposium ELMAR, 2006, Zadar, Crotia, 07-09, June 2006, pp. 9598

[12] P.Y. Simard, H..S..Malvar, J Rinker and E. Renshaw, A Foreground/Background Seperation Algorithm for Image Compression, In Data Compression Conference, 2004, pp. 498-507

[13] R.C. Gonalez, R.E. Woods, Digital Image Processing, $2^{\text {nd }}$ Edition, Addison Wesley, 2002

[14] Steven W. Smith, The Scientist and Engineer's Guide to Digital Signal Processing , $2^{\text {nd }}$ Edition, 1999

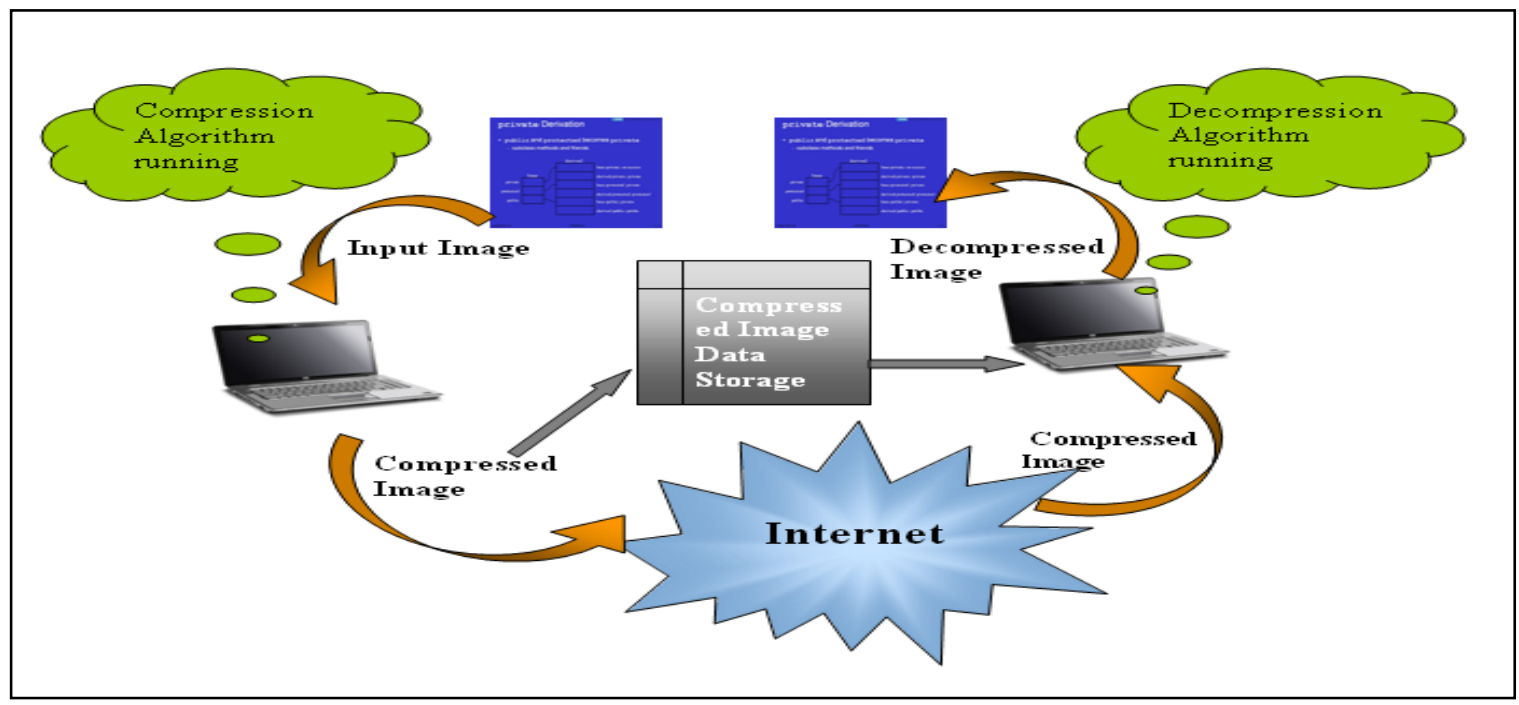

Fig 10: Workflow of our Proposed Real Time Image Codec System 